\title{
Cribriform-morular variant of papillary thyroid carcinoma and its association with familial adenomatous polyposis
}

\author{
Alejandro Perez, $B S^{a} \mathbb{D}$, Sarah Findeis, $\mathrm{MD}^{\mathrm{b}} \mathbb{D}$, Atin Agarwal, $\mathrm{MD}^{\mathrm{b}}$, Julia Berry, $\mathrm{MD}^{\mathrm{b}}$, and Katie King, $\mathrm{MD}^{\mathrm{b}}$ \\ ${ }^{a}$ Texas A\&M University College of Medicine, Bryan, Texas; ${ }^{b}$ Department of Pathology, Baylor University Medical Center, Dallas, Texas
}

\begin{abstract}
The cribriform-morular variant of papillary thyroid carcinoma (CMV-PTC) is an uncommon variant of papillary thyroid carcinoma. CMV-PTC can be associated with familial adenomatous polyposis (FAP), an autosomal-dominant polyposis syndrome caused by a mutation in the APC gene that leads to a disruption of the Wnt/beta-catenin pathway. Understanding the relation between CMVPTC and FAP is a diagnostic tool for both pathologists and clinicians, because FAP has several implications for patients and their families.
\end{abstract}

KEYWORDS Cribriform-morular; familial adenomatous polyposis; papillary carcinoma; thyroid neoplasms

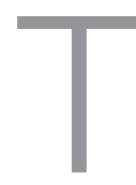

he cribriform-morular variant of papillary thyroid carcinoma (CMV-PTC) accounts for approximately $0.2 \%$ of all papillary thyroid carcinomas (PTCs). In 1990, Chan and Loo first reported the PTC variant in a patient located in Hong Kong. ${ }^{1}$ CMV-PTC occurs almost exclusively in women, with a median age of presentation of 24 years old, and is more common in the Asian population. ${ }^{2}$ Approximately $40 \%$ of patients with a CMVPTC diagnosis have familial adenomatous polyposis (FAP). ${ }^{2,3}$ Patients with CMV-PTC have a low incidence of lymph node metastases and cancer recurrence. ${ }^{2,4}$ The longterm prognosis for most cases of CMV-PTC is excellent; however, certain variants such as tall cell, columnar, and diffuse sclerosing have a worse prognosis. ${ }^{5}$ For sporadic cases, total thyroidectomy or lobectomy is an appropriate surgical treatment, whereas for FAP-associated CMV-PTC, total thyroidectomy is recommended but modified neck lymph node dissection is not necessary. ${ }^{5}$

\section{CASE 1}

A 32-year-old Asian woman with no significant past medical history presented with a $1.5-\mathrm{cm}$ left thyroid nodule found incidentally by her primary care physician on routine physical examination. The patient denied any symptoms of hyper- or hypothyroidism, weight changes, or fatigue. She had no known family history of thyroid malignancies. Her thyroid-stimulating hormone level was within normal limits. Ultrasound imaging confirmed a $1.5-\mathrm{cm}$ nodule, which was heterogeneous in appearance. Fine-needle aspiration cytologic examination showed atypical epithelial cells arranged in small groups with some small follicle formation. The atypical cells had large, irregularly shaped, hyperchromatic, and crowded nuclei; some of these cells showed nuclear grooves and pseudoinclusions. The diagnosis of highly suspicious for PTC was rendered and the patient underwent a total thyroidectomy with central node dissection.

On examination, the left thyroid lobe contained a $1.0 \times 0.9 \times 0.9 \mathrm{~cm}$ light brown, subcapsular, rubbery nodule. Microscopic examination showed a $0.9 \times 1.0 \mathrm{~cm}$ wellcircumscribed neoplasm consisting of atypical cells arranged in a cribriform pattern with a focal papillary component with scattered morules. The nuclei were crowded with irregular nuclear contours with nuclear grooves and scattered pseudonuclear inclusions. Immunohistochemical staining for beta-catenin showed diffuse nuclear and cytoplasmic staining within the neoplasm (Figure 1), and Bcl-1/Cyclin-D1 was positive in the tumor except for the morules. Estrogen receptor, progesterone receptor, and thyroid transcription factor 1 were positive in tumor cells but spared the morules, which were preferentially positive for CD10. These findings are consistent with CMV-PTC.

Corresponding author: Alejandro Perez, BS, Texas A\&M University College of Medicine, 2901 Indiana Street, Dallas, TX 75226 (e-mail: aperezdavila92@gmail.com)

Color versions of one or more of the figures in this article can be found online at www.tandfonline.com/ubmc.

Received March 9, 2019; Revised May 3, 2019; Accepted May 6, 2019. 


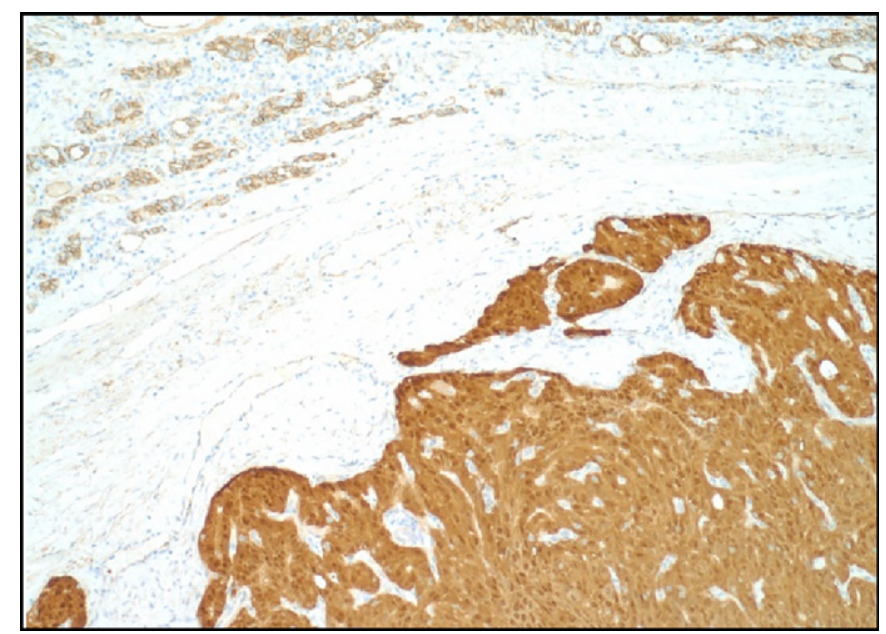

Figure 1. Case 1. Beta-catenin immunostain showing strong nuclear and cytoplasmic staining in tumor cells. The background nonneoplastic thyroid is in the upper portion of the picture for staining comparison.

\section{CASE 2}

A 19-year-old Hispanic woman with a past medical history of FAP presented with a goitrous enlargement of the right thyroid lobe, which had been present for about 2 years. Her family history was significant for FAP in her brother, father, and paternal grandfather. Although there was a family history of colon cancer, there was no family history of thyroid cancer. Her physical examination was unremarkable except for a firm palpable $5-\mathrm{cm}$ mass located in the right thyroid lobe. Laboratory findings were significant with a microcytic anemia, and thyroid-stimulating hormone levels were consistent with a euthyroid state. Ultrasound examination showed a large right thyroid nodule that contained few cystic areas. Fine-needle aspiration cytologic examination revealed sheets of cohesive follicular cells with coarse chromatin along with scattered cells with nuclear grooves and prominent nucleoli. Rare cells with nuclear pseudoinclusions were appreciated as well. Fine-needle aspiration was suspicious for PTC, and a total thyroidectomy was performed.

In the right thyroid lobe, there was a $6.9 \times 4.5 \times 3.5 \mathrm{~cm}$ well-circumscribed lesion with a tan-yellow, soft, friable, hemorrhagic cut surface. The tumor was grossly contained to the thyroid. The histologic and immunohistochemical features were identical to those of case 1 (Figure 2), and a diagnosis of CMV-PTC was rendered. The patient underwent postoperative radiotherapy and is without recurrence 16 months later.

\section{DISCUSSION}

Whenever CMV-PTC is diagnosed, additional workup is recommended because of the strong association with FAP. Although CMV-PTC is classically associated with FAP, it can also occur as a sporadic neoplasm: in a large study of patients with CMV-PTC, approximately $40 \%$ were found to have FAP, whereas the other cases were caused by sporadic mutations. ${ }^{3,5}$ Prospective recognition of CMV-PTC may allow early intervention consisting of a screening total colonoscopy, $A P C$ gene testing, and genetic counseling. ${ }^{6,7}$ Testing for the

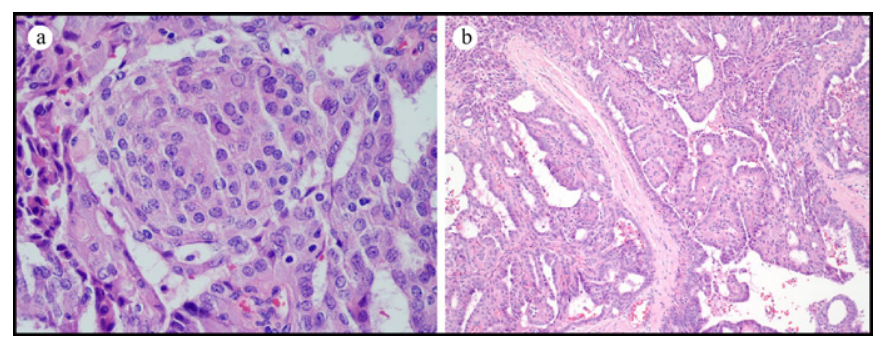

Figure 2. Case 2. (a) Morule with nuclear pseudoinclusions and nuclear grooves, high power. (b) Cribriform and papillary architecture, medium power.

$A P C$ gene would be recommended for the patient in our first case. Unfortunately, the results of genetic $A P C$ screening or colonoscopies remain unknown for both patients.

CMV-PTC usually presents before colonic manifestations in almost half of patients with FAP. ${ }^{2}$ However, in unusual cases, CMV-PTC could occur after the appearance of colonic lesions associated with FAP. Therefore, patients diagnosed with FAP should undergo long-term follow-up and be screened for thyroid nodules.

Unfortunately, clinical and radiologic findings are often nondiagnostic, and the definitive diagnosis typically is made on the resection specimen. Both patients with FAP and without FAP present with a gradually enlarging painless neck mass. ${ }^{8}$ There are no specific sonographic findings of CMVPTC, and the sonographic appearance is more like a follicular neoplasm or nodular goiter than a conventional PTC.

Histologic features and immunohistochemistry are much more diagnostic. Nuclear and cytoplasmic staining for betacatenin is the hallmark of CMV-PTC in both FAP-associated and non-FAP-associated cases. ${ }^{2,10}$ Recent studies have shown lymphoid enhancing factor 1 immunostaining to be a highly sensitive and specific marker of CMV-PTC. ${ }^{11}$

In conclusion, CMV-PTC is a rare variant of PTC. Approximately $40 \%$ of patients with CMV-PTC have FAP. Two possible chronological scenarios exist in which CMV-PTC and FAP present clinically. In the appropriate setting, the characteristic histological features, female gender, young age, and positivity of beta-catenin immunohistochemistry should lead to consideration of the diagnosis of CMV-PTC, and the diagnosis should be confirmed with fine-needle aspiration before surgery. Once CMV-PTC is diagnosed, follow-up colonoscopy and $A P C$ gene screening are recommended. Similarly, when evaluating a young patient with a history of FAP, physicians should closely monitor for thyroid nodules given the predisposition for CMV-PTC in this patient population.

\section{ORCID}

Alejandro Perez (D) http://orcid.org/0000-0002-5894-4026

Sarah Findeis (D) http://orcid.org/0000-0002-5833-0577

1. Chan JK, Loo KT. Cribriform variant of papillary thyroid carcinoma. Arch Pathol Lab Med. 1990;114:622-624. 
2. Lam A-Y, Saremi N. Cribriform-morular variant of papillary thyroid carcinoma: a distinctive type of thyroid cancer. Endocr Relat Cancer. 2017;24:R109-R121. doi:10.1530/ERC-17-0014.

3. Cameselle-Teijeiro JM, Peteiro-González D, Caneiro-Gómez J, et al. Cribriform-morular variant of thyroid carcinoma: a neoplasm with distinctive phenotype associated with the activation of the WNT/ $\beta$-catenin pathway. Mod Pathol. 2018;31:1168-1179. doi:10.1038/ s41379-018-0070-2.

4. Monappa V, Kudva R. Cytomorphologic diversity of papillary thyroid carcinoma. J Cytol. 2017;34:183-187. doi:10.4103/JOC.JOC_164_16.

5. Akaishi J, Kondo T, Sugino K, et al. Cribriform-morular variant of papillary thyroid carcinoma: clinical and pathological features of 30 cases. World J Surg. 2018;42:3616-3623. doi:10.1007/s00268-018-4644-4.

6. Andrici J, Gill AJ, Hornick JL. Next generation immunohistochemistry: emerging substitutes to genetic testing? Semin Diagn Pathol. 2018; 35:161-169. doi:10.1053/j.semdp.2017.05.004.
7. Perea Del Pozo E, Ramirez Plaza C, Padillo Ruiz J, Martos Martínez JM. Cribiform variant of papillary thyroid cancer and familial adenomatous polyposis. Int J Surg Case Rep. 2015;16:192-194. doi: 10.1016/j.ijscr.2015.08.013.

8. Pradhan D, Sharma A, Mohanty SK. Cribriform-morular variant of papillary thyroid carcinoma. Pathol Res Pract. 2015;211:712-716. doi: 10.1016/j.prp.2015.04.011.

9. Fujimoto T, Hirokawa $\mathrm{M}$, Ota $\mathrm{H}$, et al. Characteristic sonographic features of cribriform papillary thyroid carcinoma for differentiation from other thyroid nodules. J Med Ultrason. 2015;42:83-87. doi: 10.1007/s10396-014-0555-7.

10. Lam A-Y, Fridman M. Characteristics of cribriform morular variant of papillary thyroid carcinoma in post-Chernobyl affected region. Hum Pathol. 2018;74:170-177. doi:10.1016/j.humpath.2018.01.006.

11. Mohindra S, Sakr H, Sturgis C, Chute DJ. LEF-1 is a sensitive marker of cribriform morular variant of papillary thyroid carcinoma. Head Neck Pathol. 2018;12:455-462. doi:10.1007/s12105-017-0873-3. 